P44 (continued)

Objective: The goal of EPIC Kids is to promote adoption of behaviors associated with a healthy weight trajectory and prevent excess weight gain while supporting growth and development in children at risk for type 2 diabetes. Objectives include making physical activity integral to life, healthy eating, and creating home environments to ensure healthy behaviors are the easy choice.

Target Audience: Children - 9-12-years-old - who have $\geq 1$ type 2 diabetes risk factors and their caregivers.

Theory, Prior Research, Rationale: Evidence-based, efficacious community interventions for diabetes prevention in youth remain a critical need. We adapted the successful adult-focused YMCA Diabetes Prevention Program (YDPP) to serve children and families at risk. Prior studies suggest effective interventions are family-centered, target diet and physical activity, and include parents.

Description: EPIC Kids is a 12-week program led by YMCA lifestyle coaches consisting of weekly interactive sessions designed to engage families in healthy eating and physical activity, help them set goals around energy balance, and teach them to structure the home environment to support healthy lifestyle behaviors.

Evaluation: Potential public health impact was evaluated using program reach, efficacy, adoption, implementation, and maintenance data collected from YMCA administrators $(n=4)$, lifestyle coaches $(n=12)$ and families $(n=47)$ using qualitative and quantitative methods. Thirty six percent of screened families were eligible to participate; participants expressed high program satisfaction $(>87 \%$ enjoyed weekly activities) and 75\% reported high likelihood of adopting recommended changes. Weekly attendance was 65\%; program sessions were implemented as planned.

Conclusions and Implications: EPIC Kids offers a community-based youth diabetes prevention program delivered by an organization located in over 10,000 U.S. communities.

Funding: $\mathrm{NIH}$

\section{P45 Social Cognitive Theory-Based Cooking Program for Student-Athletes' Healthy Food Choices and Food Preparation Self-Efficacy}

Jenna Ellis, Master's Candidate, ejenna12@gmail.com, University of Idaho, 709 South Deakin Street, Moscow, ID 83844; K. Brown, PhD, RDN, LD; S. A. Ramsay, PhD, RDN, LD; J. M. Falk, PhD

Objective: To determine the effect of Social Cognitive Theory-based cooking classes on student-athlete self-efficacy for making healthy food choices and preparing food. Target Audience: Collegiate student-athletes enrolled in a Life Skills course $(\mathrm{N}=22)$, ages 18 -22 years.

Theory, Prior Research, Rationale: The cooking classes aimed to increase student-athletes' self-efficacy by utilizing the modeling aspect of the Social Cognitive Theory and by including hands-on cooking and meal assembling experiences. Previous studies have reported a positive association between self-efficacy and health behavior change.
Description: Student-athletes attended 4, 1-hour workshops that focused on breakfast, lunch, dinner, and snacks, respectively. Each class involved food demonstrations, hands-on food preparation, and food sampling.

Evaluation: Student-athletes completed pre and post surveys, which assessed self-efficacy for making healthy food choices and preparing food. The Wilcoxon signed-rank test was used to compare student-athletes' pre and post responses. Student-athletes reported increased self-efficacy for most healthy food choices (5/7) and cooking skills (10/11), ( $<<0.5)$. Greatest improvements in median self-efficacy were reported for steaming vegetables (2 vs. 4, $\mathrm{p}<0.0001$ ), stir-frying vegetables ( 2 vs. $3.5, \mathrm{p}=0.001$ ), and baking fish ( 2 vs. 4 , $\mathrm{p}=0.001)$. Most $(86 \%)$ reported planning to make the recipes again and all were in favor of including cooking workshops in the life skills course in the future.

Conclusions and Implications: Student-athletes accepted the hands-on cooking classes and reported increased self-efficacy for making healthy food choices and preparing food following the program's completion. Further research is needed to determine the effect of these classes on student-athletes' diets.

Funding: None

\section{P46 Eat.Right.Now. High School Curriculum for Pennsylvania SNAP-Ed Helps Philadelphia Students Eat Healthier}

JudyEnsslin, MS, RD, LDN, jae58@drexel.edu, Drexel University, 3141 Chestnut Street, Philadelphia, PA 19104; A. Marsteller, MBA, MS, RDN, LDN; S. L. Volpe, PhD, RD, LDN, FACSM

Objective: To positively impact the eating behaviors of Philadelphia high school students after receiving nutrition education utilizing the Drexel University High School Curriculum.

Target Audience: High school students from SNAP-Ed eligible schools in the School District of Philadelphia assigned to Drexel University's Eat.Right.Now. Nutrition Education Program.

Theory, Prior Research, Rationale: SNAP-Ed guidance requires the use of evidence-based nutrition education interventions. Minimal evidence-based curricula for high school students exists. These results will support the establishment of an evidence-based, high school curriculum for SNAP-Ed.

Description: High schools were randomly selected SNAPEd eligible high schools assigned to Drexel University's Eat.Right.Now. Program. The intervention utilized the modified Youth Risk Behavior Survey (YRBS). Students received a baseline survey followed by a five-lesson series using the Drexel University High School curriculum, and a post-intervention survey. A total of 1100 baseline and 972 post-intervention surveys were completed.

Evaluation: Baseline and post-intervention surveys were compared to the 2011 YRBS results for Philadelphia high school students. At baseline, intervention students demonstrated higher intakes of fruits $(p<0.0001)$, vegetables 


\section{P46 (continued)}

$(\mathrm{p}<0.0001)$, and soda $(\mathrm{p}<0.0001)$ than Philadelphia YRBS respondents. Post-intervention, these trends continued and reductions were seen in the number of students reporting no fruit $(\mathrm{p}=0.0373)$ or vegetable $(\mathrm{p}=0.0117)$ consumption. Additionally, an increase was seen in students reporting no soda consumption $(\mathrm{p}=0.0014)$.

Conclusions and Implications: Our intervention positively impacted the eating behaviors of high school students receiving this curriculum. The evidence supports this curriculum as an effective tool in helping to improve healthy eating behaviors in Philadelphia's youth and could be an effective tool for high school students throughout the nation and within SNAP-Ed.

Funding: Supplemental Nutrition Assistance Program Education

\section{P47 Child Health Assessment in the Pacific (CHAP) Summer Fellowship Program}

Marie Kainoa Fialkowski,PhD, RD, LDN, mariekf@hawaii. edu, University of Hawaii at Manoa, 2500 Campus Road, Honolulu, HI 96822; M. Sandlin, PhD;

M. Hattori-Uchima, PhD, RN, University of Guam;

M. Weichhaus, PhD, Chaminade University; P. Coleman, Northern Marianas College; R. Novotny, PhD, RDN, LDN, University of Hawaii at Manoa

Objective: Child Health Assessment in the Pacific (CHAP) Summer Fellowship program has 3 objectives: build Pacific regional capacity in early childhood nutrition and health assessment; create a network of individuals working to monitor and prevent early childhood health disparities; evaluate opportunities to sustain training in early childhood nutrition and health assessment.

Target Audience: Thirty undergraduate students from land-grant colleges and other degree seeking institutions in Hawai' $i$, the Commonwealth of the Northern Mariana Islands, Chuuk, and Guam that are enrolled in programs such as nutrition, nursing, early childhood education, and public health and committed to working in the region. Theory, Prior Research, Rationale: Few professionals in the region have the training or resources to develop, deliver, monitor and evaluate childhood health assessment programs.

Description: CHAP consists of a 6-week didactic phase followed by a 6-week application phase. In the didactic phase, students complete an introductory nutrition course and a health assessment course adapted for the Pacific online followed by a one-week hands-on training. In the application phase, students conduct a place-based miniproject. Each student will be matched with a mentor.

Evaluation: CHAP will be evaluated based on the context, input, process, and product (CIPP) model for evaluation. Quantitative data focuses on content competencies and implementation processes while qualitative data focuses on implementation process, participant and stakeholder perceptions, and overall value.

Conclusions and Implications: CHAP has the potential to contribute to a regional effort to train an interdisci- plinary team of professionals in diverse fields that will support childhood health assessment and monitoring in the Pacific.

Funding: USDA

\section{P48 B.A.L.A.N.C.E.: Evaluating the Effectiveness of a 14-Week Worksite Wellness Program on Reducing Metabolic Syndrome Risk Factors}

Melissa Manni, MS,mmanni@okstate.edu, Oklahoma State University, 921 E Echo Mountain Drive, Stillwater, OK 74075; E. Lohrman, MS, RDN; G. Gates, PhD

Objective: To determine how successful the 14-week B.A.L.A.N.C.E. program was in reducing risk factors of metabolic syndrome, and determine if participants viewed this program as helping build a healthy lifestyle.

Design, Setting, Participants, and Intervention: Eighty-nine university employees at risk for metabolic syndrome participated in the program between 2011-2014. Participants received three individual counseling sessions with an $\mathrm{RD}$, attended $\geq 10$ nutrition education sessions, and had access to a personal trainer for $\geq 60$ minutes/ week. After the 14-week intervention, participants attended $\geq 5$ monthly group meetings until the one-year mark. Participants were invited to complete a perceptions survey after completion of the program; 40 completed the survey.

Outcome Measures and Analysis: BMI, abdominal girth, cholesterol, triglycerides, HDL and LDL cholesterol, blood pressure (BP), and blood glucose were measured at week one, week 14 and one year follow-up. Fitness was assessed at baseline and week 14 using paired t-tests.

Results: Paired t-tests showed significant reductions in BMI, girth, triglycerides, blood glucose, systolic and diastolic BP, flexibility, muscular strength, and VO2 Max after 14 weeks. From baseline to one-year, paired t-tests found significant reductions in BMI, girth, blood glucose, and systolic BP. Most participants agreed or strongly agreed that participation was beneficial, they would recommend this program to other employees, and they understood more about nutrition and exercise.

Conclusions and Implications: Results show a shortterm worksite wellness program was effective in reducing metabolic syndrome risk factors, and participants perceived the program as being beneficial to their health. Funding: None

P49 Who is Aware of and Has Tried to Use MyPyramid? An Examination of Variables From the Most Recent NHANES Consumer Behavior Phone Follow-Up Module-Child (FCBS).

Erin Green, RD, emg232@cornell.edu, Cornell University, 417 Savage Hall-Division of Nutritional Sciences, Cornell University, Ithaca, NY 14850; A. Gaines, PhD;

J. Dollahite, PhD 